\title{
HEALTHY WORKING LIVES IN HEALTHY BUSINESSES: NEW OSH STRATEGY FOR SMALL ENTERPRISES?
}

\author{
Milan Tuček \\ Institute of Hygiene and Epidemiology, First Faculty of Medicine, Charles University in Prague and General University Hospital in Prague, Czech \\ Republic. E-mail: milan.tucek@lf1.cuni.cz
}

Occupational safety and health (OSH) is an area concerned with protecting the safety, health and welfare of people engaged in work or employment. Successful and productive companies around the world look after the safety and health of their workers and follow certain principles of good practice for the prevention of occupational accidents and diseases. Massey University of New Zealand hosted in February 2013 interdisciplinary conference "Understanding small enterprises" (USE Conference). Conference had a dual aim of improving both well-being of the worker (healthy working lives) and equally of the business (healthy business). This contribution represents commentary on the Second Global Interdisciplinary Conference "Understanding small enterprises" in Nelson, New Zealand, 19-22 February 2013.

There are more definitions of small businesses, one of them defines it as those which employ fewer than 20 staff. Small businesses are a substantial majority of the businesses in every major industrial sector, including dangerous sectors such as forestry, fishing, agriculture, construction, and mining (in 2007, $89 \%$ of U.S. firms had less than 20 employees and $79 \%$ had less than 10). Several studies suggest that workers in smaller businesses endure a disproportionate share of the burden of occupational injuries, illnesses and fatalities. Evidence indicates that resource deficiency, isolation, low probability of inspection, and innacurate perceptions of illness and injury incidence rates because of low frequency reduce motivation to engage in prevention activities among owner/operators in small enterprises. Thomas R. Cunningham et al. (NIOSH, United States of America) presented advancing research on delivering workplace health and safety to small businesses. In the United States, the National Institute for Occupational Safety and Health (NIOSH) is the federal agency responsible for conducting research and making recommendations for the prevention of work-related injury and illness. The NIOSH Small Business Assistance and Outreach Program is working toward the development and evaluation of a model for reaching small businesses with workplace health and safety information and practical tools for occupational safety and health programme development. Research on the unique characteristics of small businesses that affect safety and health activities has been unfocused and largely descriptive. The author underlined two critical areas which must be addressed to move small business OSH research forward: small-business construct explication issues and intervention marketing from a social-systems perspective.

Occupational risks are covered by social security legislation in European countries. Hans-Juergen Bischoff (the International Social Security Association ISSA, Machine and System Safety Section, Germany) presented the importance of return to work and prevention of occupational risks on examples from Europe with focus on small businesses. The kind of insured events and extent/range of benefits may be quite different, and, depending on the kind of event, the number of responsible in- surers may also differ. Prevention, rehabilitation, compensation represents a structure that usually makes the responsible body to invest particularly in prevention. This is the best chance to focus on selected 1) target groups, normally at high risks: branches/sectors with a high accident rate or high health risks (for example construction, agriculture, nurses) or 2) groups of workers (young and inexperienced). Losses resulting from occupational accidents and occupational diseases amount to about $4 \%$ of gross national product. In a world with a growing need for qualified workers also the potential loss, temporary or permanent, of such people needs to be tackled for social and economic reasons. New ISSA guidelines "Return to Work" and "Workplace Health Promotion" are based on the experiences of ISSA-members. The ISSA-Special Commission on Prevention with its 13 international Sections has decided to plan and put to work a working programme specifically for small enterprises. This was mainly due to similar facts worldwide: the high number of small enterprises (about $90 \%$ of all enterprises) and their limited resources. The first international project was aimed at young and inexperienced workers because they have accident rates at least two times higher than the average working population. The website also includes a self-assessment for small businesses, based on the keys to success.

10 keys to success (according to ISSA, the International Social Security Association)

\section{Healthy companies need healthy workers}

Preventing occupational injuries and diseases improves not only the performance of individual workers but also the competitiveness of a company.

\section{Better act than react}

The absence of a workplace incident does not mean that there is no risk or danger and it is far better to be preventive than reactive.

\section{Show active commitment as the owner/manager}

Visible commitment of the owner/manager is an essential condition for development of a health and safety culture within a company.

\section{Engage everyone}

Health and safety is everyone's business.

Involving employees and decision makers provides the best chance for success.

\section{Good working conditions pay}

Decent hygiene and good working conditions are cost-effective investments.

\section{Inform all persons concerned}

Information and training on workplace hazards and risks is essential for creating a safe and healthy workplace.

\section{Communicate internally with each other}

Internal communication is a part of effective prevention; to disseminate information is essential for improving workplace health and safety. 


\section{Learn from workplace incidents}

All incidents should be discussed, analyzed and reviewed in order to improve prevention practices in the workplace.

\section{Eliminate hazards/risks at the source}

Using comprehensive, integrated protection is always more effective than just the use of personal protective equipment.

\section{Keep abreast of the changes}

In the field of occupational health and safety the owner/manager must also keep abreast of the changes in legislation and in prevention practices.

Research and practice have gained considerable knowledge about small enterprises during the last decade. There is now a good understanding of the special features of small enterprises such as the owner-manager as the focal point of the organisation, the close social relations, the short distance from decission to action and not least the limited resources. The ownermanagers believe that health and safety has to be cared for because of moral and legal obligations but also that health and safety is a hassle which is costly and turns the attention away from the daily fight for survival of the business. The belief that support extended to small enterprises just means a question of scaling down large enterprise programmes to something which would look more simple, seems to be worn out (Peter Hasle, Centre for Industrial Production, Aalborg University Copenhagen). Although there is a need to be more systematic in the design of support programmes, there are also a growing number of examples of succesful support strategies which build on the understanding of small enterprises adapted to the local, sector and national context (incentive systems, networking and action learning programmes). However, working environment programmes are in many cases still placed in a sidecar with limited integration into business strategy and operation. Government programmes are most often separated in either working environment and business development.

Therefore, there is a need for the development of strategies which can overcome the split. One possibility is to prove the business case of working environment investment, and though helpful, small enterprises rarely make such investment calculations. For most owner/managers the decisive questions have to do with the practical problem the change (whether in equipment or organisation) is supposed to solve, whether the cost is sufficiently low, and whether the practical implemention and operation can be carried out with limited use of the scarce resources. The integration of business and the working environment is therefore a question of considering the two issues together in the daily operation. For instance, in construction undertaking it is a question of including the working environment in the tendering process and to use the most safe equipment because it is also the most efficient equipment.

Theresa Holizki and Rosebelle McDonald (Industry \& Labour Services, Workers' Compensation Board of British Columbia, Richmond, British Columbia, Canada) and Faith Gagnon (Gagnon Research Associates, Surrey, British Columbia, Canada) presented that there are consistent reports of discrepancies in injury and fatality rates between small and larger businesses. They undertook this study to identify patterns of safety behaviour that might explain the differences. They explored the database of the Workers' Compensation Board of British Columbia (WCB) for data on all traumatic fatalities for the period 2003-2007. Investigators' reports, coroners' reports, and employers' reports were also reviewed to determine underlying patterns of cause of fatalities in different industries and to assess whether there were significant differences between fatalities in small and larger businesses. There were 422 traumatic fatalities: 243 in small business and 179 in larger businesses. Fatality rates were 9.7 and 2.7, respectively. Factors for which there were significant differences by employer size were: fatalities within 1 month of employment (higher in small business); fatality rates in primary industries and transportation (higher in small business); vehicular incident fatality rates (higher in small business); and seat belt use (lower in small business). The incidence of alcohol abuse or recreational drug use contributing to the fatality did not differ by business size. Other findings were that cannabis use is prevalent in the logging industry, alcohol abuse is prevalent in the fishing industry, general construction workers do not always wear the necessary fall protection, and seat belt use is not prevalent among truckers and log haulers. In British Columbia, small businesses have a significantly higher traumatic fatality rate than do larger businesses. Prevention strategies are needed to address training and supervision of new workers in small businesses, recreational drug and alcohol use, and seat belt use.

Milan Tuček (the Institute of Hygiene and Epidemiology, First Faculty of Medicine, Charles University in Prague, Prague, Czech Republic) and Jana Hlávková (the National Institute of Public Health, Prague, Czech Republic) presented working risk factors and musculoskeletal disorders (MSDs) in the Czech Republic where, as in the most European countries, the musculoskeletal disorders (MSDs) represent a major problem. Their frequency has been steadily increasing to the degree that they currently rank as the second most frequent cause of temporary working disability. The MSDs represent $48 \%$ (607 cases) of the officially recognized occupational diseases (incidence 30.3/100,000 employees) and were the most frequent category of occupational diseases in the Czech Republic in 2011, with carpal tunnel syndrome being the most frequent occupational disease of them (the Czech National Registry of Occupational Diseases). The authors presented 10 years data from the Czech Republic official national system of hazard identification and risk/exposure assessment ("Categorization of working operations") used as a basic tool for effective risk management in enterprises (database of 74,731 subjects/ enterprises in 2011). Working population in the Czech Republic currently totals about 4.5 million people. Of them, about 117 thousand are exposed to vibrations ( $\approx 2.6 \%$ of total work force), about 739 thousand are exposed to overload by physical work $(\approx 16 \%$ of total work force), and about 600 thousand are working in a bad working posture $(\approx 13 \%$ of total work force). It may be therefore concluded that almost $30 \%$ of the Czech total work force is exposed to a risk factor for MDSs.

The key pertinent enactments (Act No. 258/2000 Coll. on the Public Health Protection and the amended new Labor Act No. 262/2006 Coll.) created basic legislative framework enabling to tackle the work risk factors which are undoubtedly among the important causes of the unfavourable MSDs increasing trend in the working population. These enactments prescribe employers' obligation to prepare the proposal that must comprise a comprehensive assessment of potential health risks associated with work and working conditions, results of objective measurements of intensity of these factors (4,391 measurements/exposure assessments of risk factors associated with potential MSDs in 2011), 
numbers of employees working under individual categories (males and females), and the ways of ensuring health protection. The goal of the "Categorization" is to get objective and comparable data for risk assessment, optimalization of working conditions, rational measures, and handling problems.

There have been methods for measurement and evaluation of individual factors elaborated and unified criteria established.

Although the system of categorization of working operations was prepared for all enterprises there are special situation, needs and expectations in small and medium size enterprises (SMEs). The Czech national system of categorization of working operations should be adapted or modified more to the real situation in SMEs because it was created especially for the stable workplaces and doesn't entirely reflect changeable character of work in small businesses. SMEs are rarely covered by occupational health services (OHSs) because of the costs of services ensuing for the employers, the lack of providers of these services or an unfavourable geographic location of the enterprise. There are still differences in coverage by OHSs between workers in SMEs on the one hand, and larger companies on the other. The need for OHSs in SMEs and among the self-employed is more urgent than in big enterprises and the provision of OHSs in SMEs is more dependent on the individual initiative of employees and employers than in large companies. The high rate of turnover in SMEs makes the problem still more difficult. Authors emphasize consultative role of OHSs in the hazard identification and risk/exposure assessment especially for SMEs.

Helena Palmgren et al. (the Finnish Institute of Occupational Health, Helsinki, Finland) introduced a new model for good occupational health and safety (OHS) practice for small enterprises employing less than 20 employees. The model was developed in collaboration between the Finnish Institute of Occupational Health, The Ministry of Social Affairs and Health in Finland, Social Insurance Institution and the labour parties. The model describes the process of good OHS practice in small enterprises in terms of collaboration between OHS professionals and the representatives of the organization to achieve better health and work ability of the members of the small enterprise. Occupational Health Services (OHS) is the central service system promoting the health and work ability of the working population and preventing work related illnesses and injuries in Finland. It is also the main device for prolonging working careers of the ageing population. Health, work ability and well-being at work have been shown to be positively related to productive work and the success of enterprises. The main objectives of OHS are stated in the Occupational Health Care Act (1383/2001) as follows: The employer, the employee and the OHS provider should in co-operation 1) prevent work-related illnesses and accidents, 2) improve the level of health and safety of work and the work environment, 3) maintain and improve the health, work ability and functional capacity of employees at different stages of their work careers and 4) promote the functioning of the work communities and organizations.

These topics are just a sample from the international USE conference of specialists in various fields and document the common will to find practical and viable solution for the practice of health and safety at work in small businesses. The first conference was held in Denmark in 2009 with focus on Understanding small enterprises. The focus of this second USE conference was on transforming "Understanding" into "Action" in a practical way, shortly: Transforming our understanding of small enterprises into practise to create healthy working lives in healthy businesses.

Supported by the scientific programme PRVOUK P25/LF1/2 of Charles University in Prague, Czech Republic. 